\title{
$A b$ initio quantum-enhanced optical phase estimation using real-time feedback control
}

\author{
Adriano A. Berni', Tobias Gehring1', Bo M. Nielsen1, Vitus Händchen², Matteo G. A. Paris ${ }^{3}$ \\ and Ulrik L. Andersen ${ }^{1 \star}$
}

\begin{abstract}
Optical phase estimation is a vital measurement strategy that is used to perform accurate measurements of various physical quantities including length, velocity and displacements ${ }^{1,2}$. The precision of such measurements can be greatly enhanced by the use of entangled or squeezed states of light as demonstrated in a variety of different optical systems ${ }^{3-8}$. Most of these accounts, however, deal with the measurement of a very small shift of an already known phase, which is in stark contrast to $a b$ initio phase estimation where the initial phase is unknown9-12. Here, we report on the realization of a quantum-enhanced and fully deterministic $a b$ initio phase estimation protocol based on real-time feedback control. Using robust squeezed states of light combined with a real-time Bayesian adaptive estimation algorithm, we demonstrate deterministic phase estimation with a precision beyond the quantum shot noise limit. The demonstrated protocol opens up new opportunities for quantum microscopy, quantum metrology and quantum information processing.
\end{abstract}

Parameter estimation is an integral part of any physical experiment. In some cases, the parameter under interrogation can be measured sharply and thus the uncertainty associated with the measurement is solely governed by the fluctuations of the parameter itself. In other cases, a sharp, canonical measurement cannot be realized even in principle. The optical phase is an example of such a parameter ${ }^{13}$. Because of the immense importance of performing accurate phase measurements in imaging, metrology and communications applications, numerous theoretical proposals for designing optimized phase measurements have been put forward. The basic aim is to devise a scheme that achieves the sharpest probability distribution for the phase measurement given a fixed amount of resources.

Quantum estimation theory provides the ultimate bounds on the variance of such a probability distribution ${ }^{14,15}$ in the form of the Cramer-Rao theorem ${ }^{16,17}$ : given $N$ probe states, the variance of any unbiased estimator $\hat{\phi}$ is bounded from below by the quantities

$$
\Delta^{2} \hat{\phi} \geq \frac{1}{N F(\phi)} \geq \frac{1}{N H}
$$

where $F(\phi)$ is the Fisher information (FI), which is a measure of the phase information associated with a certain detection strategy, and the quantum Fisher information (QFI) $H$ is the maximized FI over all possible detection strategies. The ultimate lower bound on the variance of $\hat{\phi}, 1 / N H$, is called the quantum Cramer-Rao (QCR) bound and $1 / N F(\phi)$ is known as the Cramer-Rao (CR) bound.
Using coherent states of light, the QFI is proportional to the mean number of photons, $H=4\langle n\rangle$, and thus the QCR bound is given by $V=1 / 4 N\langle n\rangle$, the so-called shot noise limit. This limit is superior to the standard quantum limit (SQL), which is given by $V=1 / 2 N\langle n\rangle$ and realized with a heterodyne detector ${ }^{18}$. The SQL has been surpassed in previous experiments using adaptive measurements of a coherent state ${ }^{19,20}$.

Using non-classical resources, the estimation sensitivity can be greatly enhanced beyond the shot noise limit and eventually reach the optimal Heisenberg scaling for which $V \propto 1 / N\langle n\rangle^{2}$. One class of quantum states of particular interest is the class of Gaussian states as they are relatively easy to produce and comparatively robust against $\operatorname{losses}^{21}$. It has been shown theoretically that by employing pure Gaussian squeezed states, the shot noise limit can be surpassed and the QCR bound $(V=1 / 2 N \sinh (2 r)$, where $r$ is the squeezing parameter) can be asymptotically approached by means of simple homodyne detection, Bayesian estimation and optical feedback ${ }^{22,23}$. In this Letter, we demonstrate such a squeezing-enhanced quantum phase algorithm.

The phase estimation protocol is schematically shown in Fig. 1a. A squeezed state of light acquires an unknown phase shift within the interval $[0, \pi / 2]$, which is subsequently estimated using homodyne detection and Bayesian inference. Note that this is different from the standard two-mode Mach-Zehnder interferometer. Using such a measurement, the FI reaches the QFI only for one specific phase shift (corresponding to $\phi_{\mathrm{opt}}^{\mathrm{th}}=\arccos (\tanh (2 r)) / 2$ ), and estimation beyond the SQL can be realized only in a limited phase interval as illustrated in Fig. 1b. This is sufficient for quantum phase sensing (where a tiny shift of a known phase is measured), but not for ab initio phase estimation. To circumvent this limitation, the idea is to implement an adaptive feedback scheme such that the system is driven towards the optimal phase. More specifically, our strategy is to detect a small fraction of the available samples, use Bayesian inference to obtain a first rough estimate of the phase, use that information to shift the local oscillator towards the optimal phase point, and finally measure the remaining samples to deduce the final estimate via Bayesian inference. As an alternative, the feedback can be continuous, but the two-step adaptive approach is sufficient to asymptotically reach the QCR bound ${ }^{24}$.

Bayesian inference provides a framework for deducing the a posteriori probability distribution (PPD) of the phase conditioned on the $M$ sampled homodyne data $\{x\}_{M}$ :

$$
P\left(\phi \mid\{x\}_{M}\right) \stackrel{M \gg 1}{\simeq} \frac{1}{\mathcal{N}} \prod_{i=1}^{M} P\left(x_{i}, \phi\right)^{M P\left(x_{i}, \phi\right)}
$$

where $\mathcal{N}$ is a normalization constant. $P\left(x_{i}, \phi\right)$ are the individual 

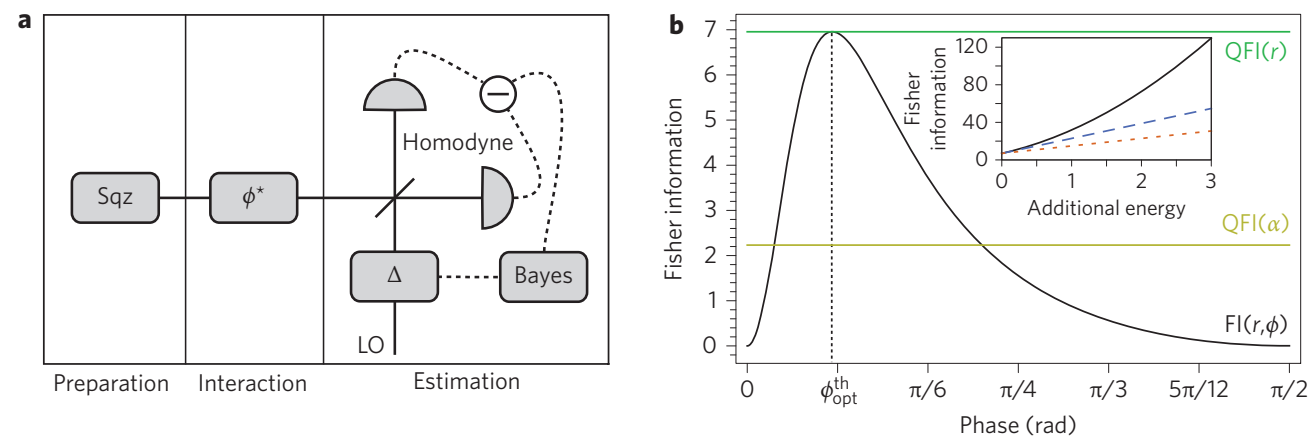

Figure 1 | Principle of squeezing-enhanced phase estimation. $\mathbf{a}$, The input squeezed vacuum state (denoted sqz) accumulates a phase shift $\phi^{\star}$, which is measured using homodyne detection, real-time Bayesian inference and feedback. The feedback is applied to the local oscillator of the homodyne detector to drive the measuring phase towards the phase that maximizes the Fisher information $(\mathrm{FI}), \phi_{\mathrm{opt}}^{\mathrm{th}}$. $\mathbf{b}, \mathrm{Fl}$ as a function of phase for a pure $6 \mathrm{~dB}$ squeezed vacuum state. Horizontal lines represent the quantum Fisher information (QFI) for a similarly pure squeezed state (green) and for a coherent state (yellow). Inset: $\mathrm{Fl}$ at $\phi_{\mathrm{opt}}^{\text {th }}$ as a function of additional energy (in terms of average photon number) starting with a $6 \mathrm{~dB}$ pure squeezed state. The energy addition processes are squeezing (black solid line), displacement (blue dashed) and noise addition onto the anti-squeezed direction (red dotted).

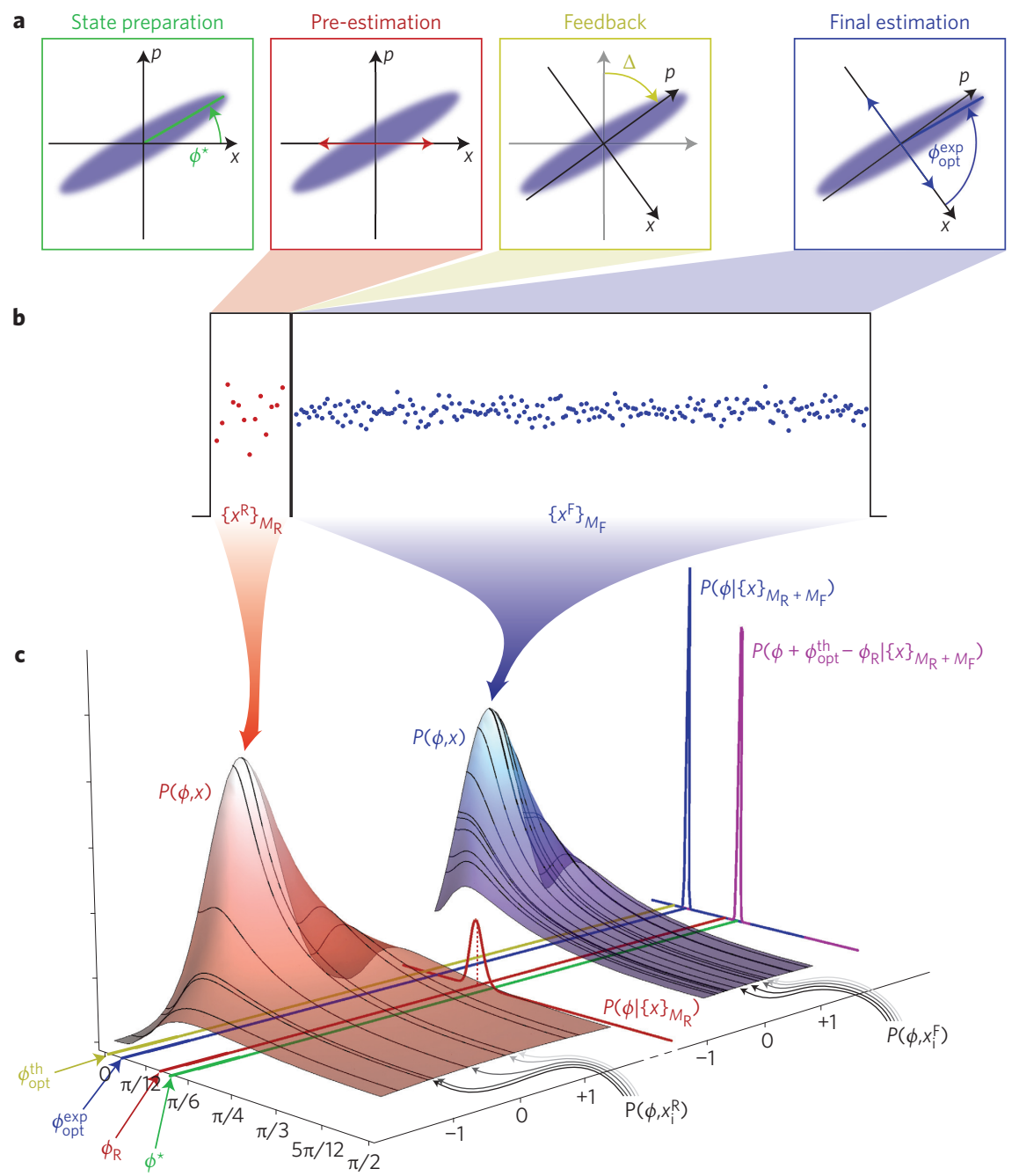

Figure 2 | Working principle of the Bayesian feedback scheme. a, Phase-space representation of the different steps of the protocol: state preparation of a squeezed state (green) with an unknown phase shift $\phi^{\star}$; pre-estimation of the phase shift via Bayesian inference by performing $M_{R}$ homodyne measurements of the $x$ quadrature (which yields the value $\phi_{R}$ ); based on the pre-estimate, rotation of the squeezed state in phase space via feedback; a second homodyne detection stage, in which $M_{\mathrm{F}} \gg M_{R}$ quadrature data are collected to obtain the estimate $\phi_{\mathrm{opt}}^{\text {exp }}$. $\mathbf{b}$, Illustration of a subset of the acquired quadrature data during pre- and final estimation. The complete set is shown in Fig. 3. c, Illustration of marginal distributions and PPDs for the different steps. Each homodyne sample $x_{i}$ collected during the estimation stages is used to compute a marginal phase distribution $P\left(\phi, x_{i}\right)$. A subset of such distributions is shown by the black curves. A complete set will cover the red and blue surfaces. The distributions are multiplied according to equation (2) to obtain the PDD $P\left(\phi \mid\{x\}_{M}\right)$ for the rough (red curve) and final (blue and purple curves) estimations. 


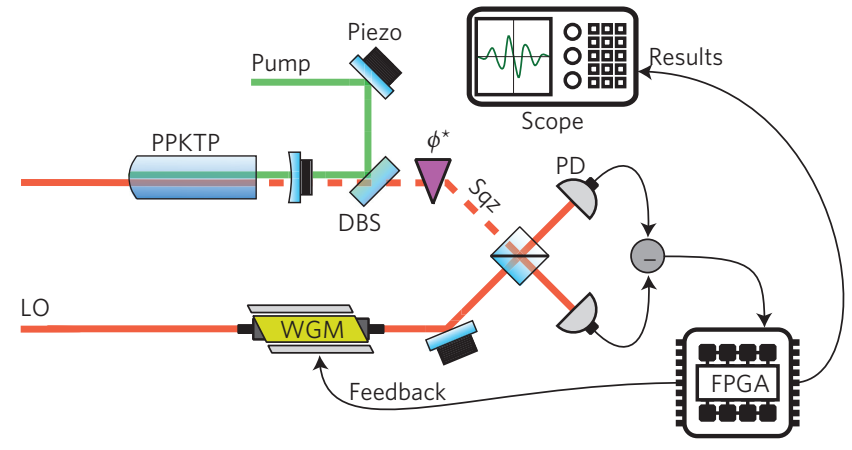

Figure 3 | Simplified experimental layout. Squeezed vacuum states are generated by degenerate parametric downconversion using a periodically poled (PP) KTP crystal inside a cavity formed by an external mirror and the curved end-facet of the crystal. The process is driven by a pump beam at $775 \mathrm{~nm}$ producing squeezed light at sidebands around the carrier of 1,550 nm. The squeezed light is combined with a phase-controlled local oscillator (LO), detected with high-efficiency photodiodes and processed in the FPGA. DBS, dichroic beamsplitter; Sqz, squeezed light; PD, photodetector; WGM, waveguide phase modulator; FPGA, field-programmable gate array. More information about the set-up can be found in Supplementary Chapter 2.

marginal phase distributions conditioned on single homodyne measurement outcomes $x_{i}$ and are given by

$$
P\left(x_{i}, \phi\right)=\frac{1}{\sqrt{2 \pi \sigma_{\phi}^{2}}} \exp \left[-\frac{x_{i}^{2}}{2 \sigma_{\phi}^{2}}\right]
$$

where $\sigma_{\phi}^{2}=\left(2 n_{\text {th }}+1\right)\left[e^{-2 r} \cos ^{2}(\phi)+e^{2 r} \sin ^{2}(\phi)\right]$ is the variance of the probe state ( $n_{\text {th }}$ is the number of thermal photons). For a large number of homodyne samples, the PDD converges to a Gaussian distribution centred on the true value of the parameter, $\phi^{*}$, with a variance of $\left(N F\left(\phi^{*}\right)\right)^{-1}$. If $n_{\mathrm{th}}=0$, the use of homodyne detection and Bayesian inference yields operation at the QCR bound, which means that the protocol is optimal.

The behaviour of the FI while energy is added to a pure squeezed state is illustrated in the inset of Fig. $1 \mathrm{~b}$ for three different energy-adding mechanisms: (1) pure squeezing (solid black line), (2) phase space displacement (blue dashed line), and (3) incoherent noise addition into the anti-squeezed quadrature (red dotted line). It is clear that all three processes lead to an increase in the FI, but only the pure squeezing process stays at the ultimate QCR bound for Gaussian states ${ }^{25}$. In any realistic implementation, however, the squeezed state will not be pure but will be polluted by noise, and so the actual implementation will not reach the QCR bound, but instead a sub-optimal bound that we term the 'optimal Cramer-Rao' (OCR) bound ${ }^{25}$. We note that the coherent displacement operation only deviates slightly from the QCR bound for low energies and constitutes a means of resolving the phase in the range $[0,2 \pi]$.

The working principle of the Bayesian scheme combined with adaptive feedback is illustrated in Fig. 2. We assume that the input squeezed state is prepared with an unknown phase shift $\phi^{*}$. The first homodyning stage (pre-estimation) produces a list of $M_{\mathrm{R}}$ quadrature measurement outcomes (red dots), each of which is associated with a probability distribution for the phase (equation (3) and the black curves on the red surface in Fig. 2). Based on all these distributions, the PPD is computed using equation (2) and illustrated by the red curve in Fig. 2 . The rough phase estimate is then given by the maximum value, $\phi_{R}=\phi^{*}+\delta \phi_{R}$, of the distribution with an estimation error $\delta \phi_{\mathrm{R}}$. Because the optimal phase is $\phi_{\mathrm{opt}}^{\text {th }}$, we introduce a phase shift $\Delta=\phi_{\mathrm{R}}-\phi_{\mathrm{opt}}^{\text {th }}$ onto the local oscillator, thereby rotating the squeezed state phase towards the optimal phase: $\phi^{*} \rightarrow \phi^{*}-\Delta=\phi_{\mathrm{opt}}^{\mathrm{th}}-\delta \phi_{\mathrm{R}}$. The second Bayesian homodyning stage is then performed, which delivers an estimation of the new phase $\phi_{\mathrm{opt}}^{\mathrm{exp}}=\phi_{\mathrm{opt}}^{\mathrm{th}}-\delta \phi_{\mathrm{R}}+\delta \phi_{\mathrm{F}}$, based on a larger number of samples $M_{\mathrm{F}} \gg M_{\mathrm{R}}$ (blue dots). Due to the larger number of homodyne samples and the enhanced homodyne sensitivity (as a result of the proximity to the optimal phase), we obtain $\delta \phi_{\mathrm{F}} \ll \delta \phi_{\mathrm{R}}$ and a very sharp PDD (blue curve). To obtain the final estimate we add $\Delta$ to $\phi_{\mathrm{opt}}^{\exp }$ and get est $\left[\phi^{*}\right]=\phi_{\mathrm{opt}}^{\exp }+\Delta=\phi^{*}+\delta \phi_{\mathrm{F}}$, which yields the input phase $\phi^{*}$ with an error $\delta \phi_{\mathrm{F}}$ (corresponding to the purple curve).

We now turn to the experimental demonstration of the scheme, which comprises a source of squeezed light, a homodyne detector and fast feedback electronics (Fig. 3). The squeezed light source is based on cavity-enhanced parametric downconversion, and the noise suppression of one quadrature is measured as $-5.69 \pm 0.07 \mathrm{~dB}$ relative to the vacuum noise limit while the noise of the conjugate quadrature is amplified by $11.83 \pm 0.09 \mathrm{~dB}$ (Supplementary Chapter 4.1).

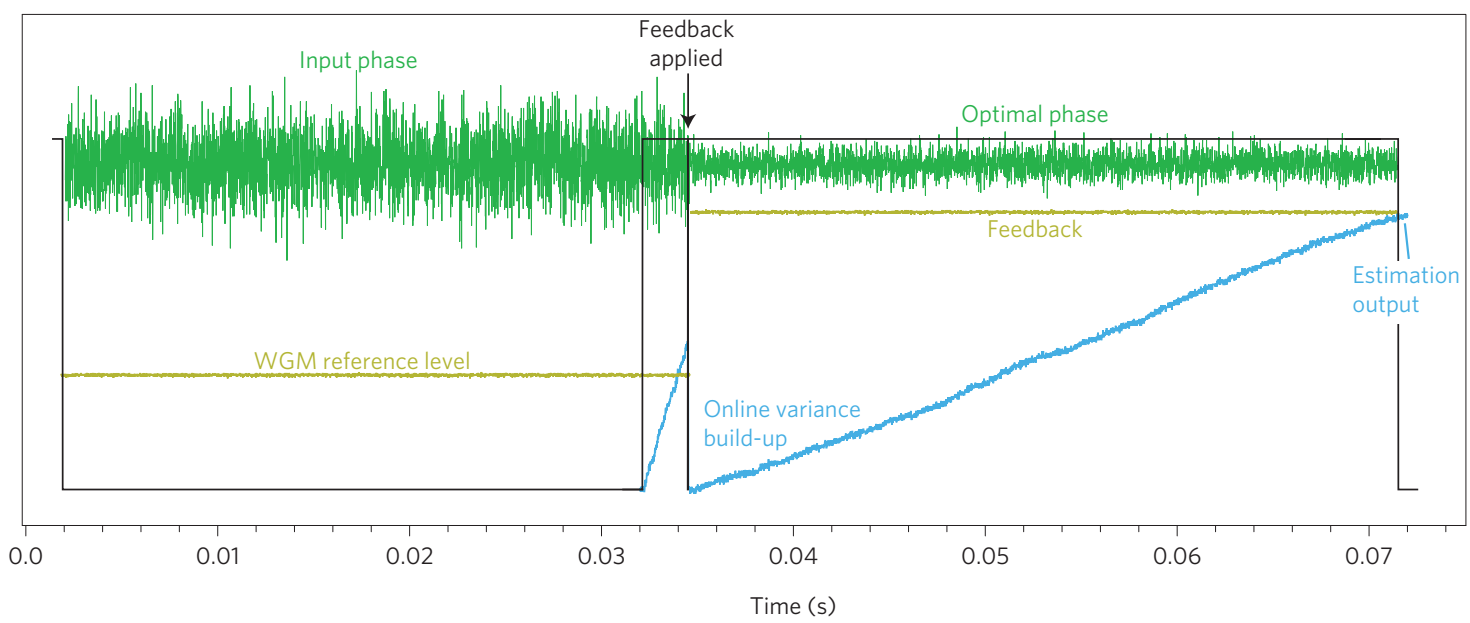

Figure 4 | Illustration of a single measurement sequence. Acquired homodyne quadrature data (green) together with the reference levels (yellow) for the WGM (which controls the relative phase) as a function of time for three different stages: preparation, rough estimation and final estimation. During the rough estimation stage the LO is still set on the input phase, so the homodyne variance is the same as during the state preparation stage. In the final estimation stage the feedback signal to the WGM is applied (black arrow), which shifts the phase of the state towards the optimal phase, resulting in a change in the spread of the homodyne data. Blue data refer to the on-line accumulation of the (unnormalized) phase variance provided by the FPGA. The final output yields the phase variance. 

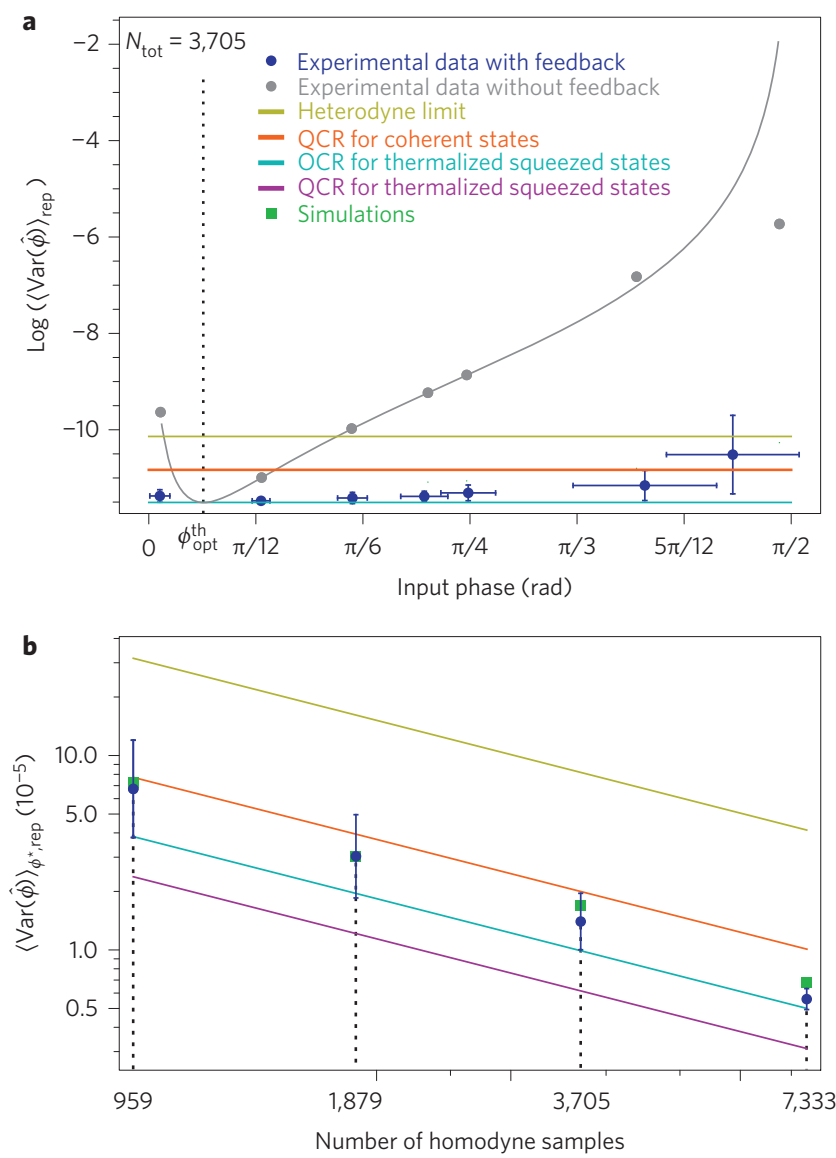

Figure 5 | Estimation variance results. a, Estimation variance versus input phase for fixed number of homodyne samples. The experimental data (dark blue) are shown for seven input phases in the $[0, \pi / 2]$ range with vertical error bars giving the standard deviations over 80 repetitions and the horizontal error bars showing the standard deviations over the slightly varying input phases over the experimental repetitions. b. Estimation variance versus number of homodyne samples. The experimental results (dark blue circles) are obtained by averaging the final estimation PPD variance over 80 repetitions of the experiment and over all tested input phases in each fixed-energy run of the experiment. Error bars indicate the statistical error over the repetitions, averaged over the input phases. Note that the definition for the variance is $\left\langle\phi^{2}\right\rangle-\langle\phi\rangle^{2}$, which is nearly identical to the Holevo variance as all the involved phase distributions are very narrow.

A high-efficiency homodyne detector is used to measure the state, the setting of which is controlled in real time by a field-programmable gate array (see Methods). An example of a measurement run is presented in Fig. 4.

To investigate the performance of the protocol for one specific input phase, we fixed the total number of homodyne samples to $N_{\text {tot }}$ and performed 80 repetitions. The division between the number of samples for pre- and final estimation was optimized by performing Monte Carlo simulations of the experiment with the fraction of the sample numbers as a free parameter ${ }^{26}$. For every $N_{\text {tot }}$ we found the optimized fraction, which was then subsequently used in the experiment.

The results of the estimation variance are shown in Fig. 5a for different input phases both with (blue symbols) and without (grey symbols) feedback control. Theoretical predictions for an ideal implementation of the two schemes given the estimated input state are also presented (cyan and grey curves), and it is clear that the experimental results are in good agreement with the theory. As expected, the protocol performs best when the input phase is in the proximity of the optimal phase (corresponding to $\left.\phi_{\mathrm{opt}}^{\text {th }}=0.132 \pm 0.001\right)$ as this will yield the best pre-estimation of the phase. This explains the small discrepancy between the data and theory as the input phase gets further away from $\phi_{\mathrm{opt}}^{\text {th }}$. It is clear that the scheme beats the heterodyne (yellow) as well as the optimized coherent state (orange) approaches.

We further investigated the variance scaling for different sample numbers $N_{\text {tot. }}$. In Fig. $5 \mathrm{~b}$ we present the results corresponding to four different sample numbers where each data point is associated with an average over several different initial phases distributed across the phase range. For example, for $N_{\text {tot }}=3,075$, the variance in Fig. $5 \mathrm{~b}$ is an average of the seven realizations presented in Fig. 5a. As the theoretical prediction for our protocol (cyan curve) assumes the asymptotic limit, the observed discrepancy is mostly pronounced for low sample numbers. We also compare the experimental results with Monte Carlo simulated data (squares), which yields near perfect agreement. The theoretical QCR bound for the coherent state (orange), the squeezed state (purple) as well as the bound for heterodyne detection (yellow) are inserted for comparison. From the figure, it is clear that all measurement points are substantially below the SQL (or heterodyne limit) and even below the QCR bound for coherent states.

In conclusion, we have implemented a real-time adaptive protocol for $a b$ initio phase estimation designed to asymptotically saturate the QCR limit beyond the shot noise limit. In contrast to a previous implementation of $a b$ initio phase estimation ${ }^{12}$, we use a source of deterministically generated squeezed states of light and real-time feedback control. We believe that our protocol can be applied to a variety of different metrological and informational tasks, in particular considering the recent advances in the production of pure and highly squeezed states of light ${ }^{27}$. For example, our developed technique can be used to image ultra-sensitive living cells ${ }^{28}$ and single molecules ${ }^{29}$ beyond the quantum limit. To empower these applications further, in the future we intend to consider feedbackbased protocols for the simultaneous estimation of conjugate quadratures $^{30}$ and full phase range estimation at the QCR bound.

\section{Methods}

Methods and any associated references are available in the online version of the paper.

Received 5 February 2015; accepted 6 July 2015; published online 10 August 2015

\section{References}

1. Caves, C. M. Quantum mechanical noise in an interferometer. Phys. Rev. D 23, 1693-1708 (1981)

2. Giovannetti, V., Lloyd, S. \& Maccone, L. Advances in quantum metrology. Nature Photon. 5, 222-229 (2011).

3. Nagata, T., Okamoto, R., O’Brien, J., Sasaki, K. \& Takeuchi, S. Beating the standard quantum limit with four-entangled photons. Science 316, 726 (2007).

4. Banaszek, K., Demkowicz-Dobrzanski, R. \& Walmsley, I. A. Quantum states made to measure. Nature Photon. 3, 673-676 (2009).

5. Kacprowicz, M., Demkowicz-Dobrzanski, R., Wasilewski, W., Banaszek, K. \& Walmsley, I. A. Experimental quantum-enhanced estimation of a lossy phase shift. Nature Photon. 4, 357-360 (2010).

6. The LIGO Scientific Collaboration. tA gravitational wave observatory operating beyond the quantum shot-noise limit. Nature Phys. 7, 962-965 (2011)

7. Yonezawa, H. et al. Quantum-enhanced optical phase tracking. Science 337, 1514-1517 (2012)

8. Hoff, U. B. et al. Quantum-enhanced micromechanical displacement sensitivity. Opt. Lett. 38, 1413-1415 (2012).

9. Wiseman, H. M. Adaptive phase measurement of optical modes: going beyond the marginal Q distribution. Phys. Rev. Lett. 75, 4587-4590 (1995).

10. Berry, D. W. \& Wiseman, H. M. Adaptive phase measurements for narrowband squeezed beams. Phys. Rev. A 73, 063824 (2006).

11. Kolodynski, J. \& Demkowicz-Dobrzanski, R. Phase estimation without a priori phase knowledge in the presence of loss. Phys. Rev. A 82, 053804 (2010).

12. Xiang, G. Y., Higgins, B. L., Berry, D. W., Wiseman, H. M. \& Pryde, G. J. Entanglement-enhanced measurement of a completely unknown optical phase. Nature Photon. 5, 43-47 (2010). 
13. Barnett, S. M. \& Pegg, D. T. Phase in quantum optics. J. Phys. A 19, 3849-3862 (1986).

14. Hradil, Z. \& Rehacek, J. Quantum interference and Fisher information. Phys. Lett. A 334, 267-272 (2005).

15. Escher, B. M., de Matos Filho, R. L. \& Davidovich, L. General framework for estimating the ultimate precision limit in noisy quantum-enhanced metrology. Nature Phys. 7, 406-411 (2011).

16. Cramer, H. Mathematical Methods of Statistics (Princeton Univ. Press, 1946).

17. Braunstein, S. L. \& Caves, C. M. Statistical distance and the geometry of quantum states. Phys. Rev. Lett. 72, 3439-3443 (1994).

18. D’Ariano, G. M., Paris, M. G. A. \& Seno, R. Feedback-assisted homodyne detection of phase shifts. Phys. Rev. A 54, 4495-4504 (1996).

19. Armen, M. A., Au, J. K., Stockton, J. K., Doherty, A. C. \& Mabuchi, H. Adaptive homodyne measurement of optical phase. Phys. Rev. Lett. 89, 133602 (2002).

20. Wheatley, T. A. et al. Adaptive optical phase estimation using time-symmetric quantum smoothing. Phys. Rev. Lett. 104, 093601 (2010).

21. Weedbrook, C. et al. Gaussian quantum information. Rev. Mod. Phys. 84, 621-669 (2012).

22. Monras, A. Optimal phase measurements with pure Gaussian states. Phys. Rev. A 73, 033821 (2006).

23. Olivares, S. \& Paris, M. G. A. Bayesian estimation in homodyne interferometry. J. Phys. B 42, 055506 (2009).

24. Barndorff-Nielsen, O. E. \& Gill, R. D. Fisher information in quantum statistics. J. Phys. A 33, 4481-4490 (2000).

25. Aspachs, M., Calsamiglia, J., Munoz-Tapia, R. \& Bagan, E. Phase estimation for thermal Gaussian states. Phys. Rev. A 79, 033834 (2009).

26. Berni, A. Quantum Metrology With Squeezed Light. Master's thesis (Università degli Studi di Milano, 2011).

27. Eberle, T., Händchen, V. \& Schnabel, R. Stable control of $10 \mathrm{~dB}$ two-mode squeezed vacuum states of light. Opt. Express 21, 11546-11553 (2013).
28. Carlton, P. M. et al. Fast live simultaneous multiwavelength four-dimensional optical microscopy. Proc. Natl Acad. Sci. USA 107, 16016-16022 (2010).

29. Pototschnig, M. et al. Controlling the phase of a light beam with a single molecule. Phys. Rev. Lett. 107, 063001 (2011).

30. Genoni, M. G. et al. Optimal estimation of joint parameters in phase space. Phys. Rev. A 87, 012107 (2013).

\section{Acknowledgements}

The authors acknowledge financial support from the Danish Agency for Science, Technology and Innovation (Sapere Aude grant from FTP: 10-081599) and the Lundbeck Foundation. T.G. was supported by the HC Ørsted Postdoc programme. The authors would like to thank Roman Schnabel from the Albert Einstein Institute in Hannover for the support in using the squeezing source.

\section{Author contributions}

A.A.B. and T.G. performed the main experiment and analysed the data. A.A.B., T.G. and B.M.N. developed the feedback protocol. T.G. and V.H. built the squeezed light source. A.A.B., T.G., M.G.A.P. and U.L.A. discussed the results. A.A.B and U.L.A wrote the paper with support from T.G. and M.G.A.P. U.L.A. conceived and supervised the project.

\section{Additional information}

Supplementary information is available in the online version of the paper. Reprints and permissions information is available online at www.nature.com/reprints. Correspondence and requests for materials should be addressed to U.L.A.

\section{Competing financial interests}

The authors declare no competing financial interests. 


\section{Methods}

A high-efficiency homodyne detector was used for measurements, including a

local (reference) oscillator transversing a fast waveguide phase modulator (WGM)

(Fig. 3). The demodulated homodyne a.c. signal was fed to a field-programmable gate array (FPGA), which was used to perform the pre-estimation based on Bayesian inference as outlined in the main text, and subsequently handled the feedback signal to the waveguide modulator. Due to the FPGA architecture, full implementation of the PPD computation was not convenient, because it would represent a speed bottleneck during the feedback stage. We therefore resorted to an equivalent formalism based on look-up tables and proper calibration (see Supplementary Chapter 4). The estimation result, the homodyne a.c. signal and the feedback signal were collected by an oscilloscope, and a typical output during a single measurement period is shown in Fig. 4. The first part of the trace represents state preparation in which the local oscillator is phase-locked to the squeezed state. Once the estimation stage started, the phase lock was released to enable the feedback of the FPGA, and after estimation, the lock was re-established to prepare for the new measurement run. Due to the high stability of the set-up, the relative phase between the local oscillator and the squeezed state did not drift within a single estimation period lasting between 10 and $70 \mu \mathrm{s}$. 